\title{
Comparative cranial osteology of subadult eucentrosauran ceratopsid dinosaurs from the Two Medicine Formation, Montana, indicates sequence of ornamentation development and complex supraorbital ontogenetic change
}

John P. Wilson and John B. Scannella

Acta Palaeontologica Polonica 66 (4), 2021: 797-814 doi:https://doi.org/10.4202/app.00797.2020

The eucentrosauran centrosaurines Einiosaurus procurvicornis and Achelousaurus horneri are the two most commonly recovered ceratopsids from the Campanian Two Medicine Formation of northwestern Montana, USA. Einiosaurus procurvicornis is known from at least 15 individuals recovered from two monospecific bonebeds, while Achelousaurus horneri is primarily known from one articulated adult cranium as well as two isolated subadult individuals previously referred to the taxon. Previous assessments of ontogeny in these taxa, alongside closely related centrosaurines, focused primarily on crania of mature individuals and disarticulated elements of immature individuals. Here we describe an articulated subadult Einiosaurus procurvicornis skull (MOR 456 8-8-87-1) from the Einiosaurus procurvicornis type locality bonebed and compare its cranial ornamental development with the only identically sized articulated subadult eucentrosauran skull from the Two Medicine Formation, MOR 591. These individuals represent the only known articulated subadult skulls from the hypothesized eucentrosauran lineage in the Two Medicine Formation, thereby enabling comparison of early ontogenetic developmental sequence and timing of all three primary cranial ornaments (nasal, supraorbital, and parietosquamosal frill). Comparison indicates that parietosquamosal frill and supraorbital ornamentation development may have preceded nasal horncore development in these taxa. MOR 456 8-8-87-1 fills a gap between the plesiomorphic morphology of juvenile Einiosaurus procurvicornis supraorbital horncores and the rounded, spheroid mass of bone which characterizes adults. The complete left squamosal of MOR 456 8-8-87-1 is of adult size, in contrast to its shorter face and immature facial ornamentation, which suggests that in Einiosaurus procurvicornis, the face and facial ornamentation development occurred after the parietosquamosal frill had reached adult size.

Key words: Dinosauria, Ceratopsia, Centrosaurine, ontogeny, Cretaceous, Two Medicine Formation, USA.

John P. Wilson [jackwilson1899@gmail.com], Varricchio Lab, Department of Earth Sciences, Montana State University, Bozeman, 
Montana, USA. John B. Scannella [john.scannella@montana.edu], Museum of the Rockies and Department of Earth Sciences, Montana State University, Bozeman, Montana, USA.

This is an open-access article distributed under the terms of the Creative Commons Attribution License (for details please see creativecommons.org), which permits unrestricted use, distribution, and reproduction in any medium, provided the original author and source are credited.

Foris Full text $(8,558.4 \mathrm{kB})$ 